\title{
TUTORIALES WEB: INDICADORES Y EJEMPLOS DE BUENAS PRÁCTICAS
}

\author{
Marta Somoza-Fernández y Concepción Rodríguez-Parada
}
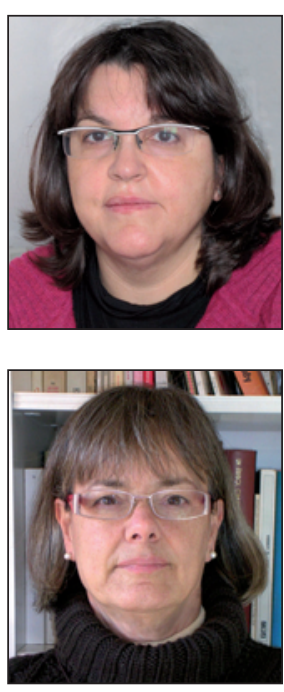

Marta Somoza-Fernández es licenciada en historia contemporánea (1989) y antropología cultural (1995) y doctora en documentación (2009) por la Universitat de Barcelona (UB). Fue responsable del Servei de Teledocumentació de l'Àrea de Ciències de la Salut de la biblioteca de la UB hasta el año 2002. Desde 1999 es profesora del Dept. de Biblioteconomia i Documentació de la UB. Sus líneas de investigación se centran en bases de datos documentales, recuperación de la información, estudios bibliométricos y formación de usuarios. Es miembro del Grup d'alfabetització informacional i formació d'usuaris (AlfinCat) del Col-legi de Bibliotecaris-Documentalistes de Catalunya.

Concepción Rodríguez-Parada es licenciada en filosofía [Universitat de Barcelona (UB) 1982], diplomada en biblioteconomía y documentación (1987, UB) y doctora en documentación (2009, UB). Desde 1993 es profesora del Departament de Biblioteconomia i Documentació de la UB. Miembro de los grupos de investigación Denea (Detecció de necessitats de l'audiència) y Exemplar, y colaboradora del proyecto Topografía de la espiritualidad femenina mendicante en Cataluña y reinos peninsulares de la Edad Media. Sus líneas de investigación se centran en la gestión de bibliotecas, la deontología, el estudio del patrimonio bibliográfico y la espiritualidad femenina medieval.

Universitat de Barcelona Dept. de Biblioteconomia i Documentació Melcior de Palau, 140 08014 Barcelona, España msomoza@ub.edu crodriguezp@ub.edu

\section{Resumen}

Las bibliotecas universitarias realizan tutoriales web sobre una gran variedad de temáticas. Muchas veces van dirigidos a la alfabetización informacional (alfin) y a mostrar a los usuarios las mejoras tecnológicas de las interfaces de consulta interactiva. Se ofrece una metodología basada en treinta y cuatro indicadores para elaborar, evaluar y mejorar los tutoriales creados por las bibliotecas de cualquier ámbito y especialidad. Se presentan adicionalmente ejemplos de buenas prácticas que ilustran la descripción del indicador y pueden servir de referencia para los servicios que deseen crear sus propios tutoriales.

\section{Palabras clave}

Tutoriales web, Bibliotecas universitarias, Indicadores de evaluación, Diseño, Buenas prácticas, Habilidades informacionales, Alfabetización informacional, Alfin.

Title: Web tutorials: indicators and best practice examples

\section{Abstract}

University libraries make web tutorials on a variety of topics. Often they are efforts to improve user's information literacy and to show them technological improvements on interactive interfaces. A methodology with a total of 34 indicators to be taken into account in the development, evaluation and improvement of library tutorials created in any area and specialty is presented, together with examples of best practices that illustrate the indicators and that can be a guideline for library services wishing to make their own tutorials.

\section{Keywords}

Web tutorials, University libraries, Assessment, Evaluation indicators, Check list, Design, Best practices, Informational skills, Information literacy.

Somoza-Fernández, Marta; Rodríguez-Parada, Concepción. "Tutoriales universitarios: indicadores y ejemplos de buenas prácticas". El profesional de la información, 2011, enero-febrero, v. 20, n. 1, pp. 38-46.

DOI: 10.3145/epi.2011.ene.05 


\section{Introducción}

Las bibliotecas universitarias han elaborado materiales de apoyo para la formación de usuarios, como, por ejemplo, guías o manuales para facilitar la consulta de catálogos o bases de datos. La evolución tecnológica ha permitido la creación de materiales multimedia o hipermedia y la elaboración de vídeos sobre recursos y servicios de la biblioteca. En este momento las bibliotecas empiezan a considerar los tutoriales como el material formativo más completo puesto que implica una organización didáctica y tecnológica más compleja que el resto de materiales. Sin embargo todos ellos presentan un mismo inconveniente: la edición y actualización de sus contenidos. Esta dificultad ha sido superada con la web, que amplía la accesibilidad y abarata la edición de contenidos. No obstante, en los últimos años se dan otros elementos, aparte de los tecnológicos, que exigen reinterpretar los materiales didácticos y revisar su diseño y contenido. En principio, los ciudadanos deben adquirir los conceptos y dominar las prácticas de la alfabetización informacional para que puedan utilizar la información de manera eficaz y ética a lo largo de toda su vida laboral y personal. Sin embargo, el ámbito de la educación y la formación presenta un panorama más complejo del proceso de aprendizaje, pues el alumno no cuenta con los mismos conocimientos previos ni aprende de la misma manera que antes. En este contexto es donde presentamos algunas recomendaciones que permitan la creación de tutoriales efectivos y adaptados.

No existen estudios de tutoriales universitarios con conclusiones representativas ni con métodos para evaluarlos

\section{Antecedentes}

En Documentación la bibliografía sobre tutoriales es escasa y mayoritariamente presenta estudios de casos que muestran el proceso de elaboración de un tutorial por parte de una biblioteca concreta.

Las publicaciones que analizan un conjunto de ellos, o bien acostumbran a llegar a conclusiones muy generales (Dewald, 1999), o sólo evalúan un aspecto particular como puede ser el de la interactividad (Tancheva, 2003), o el aprendizaje activo (Hrycaj, 2005). Un estudio reciente ha examinado una muestra representativa de 274 tutoriales de las bibliotecas médicas americanas (Anderson et al., 2008) pero nuevamente los describe desde una perspectiva muy amplia por cuanto se limita a ofrecer indicaciones generales para mejorar aspectos concretos pero sin llegar a profundizar o sistematizar la evaluación.

Desde el punto de vista de la evaluación encontramos bibliografía que trata de las valoraciones que los usuarios hacen del tutorial. Estudios como el de Donaldson (2000), Michel (2001) o Bury y Oud (2005) evalúan a través de la encuesta o la entrevista diferentes aspectos, tanto de los contenidos como la usabilidad del tutorial. Todos los autores señalan la utilidad de estos tests para detectar los puntos fuertes y aquellos otros que deben ser mejorados.
Otros trabajos evalúan los conocimientos adquiridos. Así el artículo de Bracke y Dickstein (2002) compara los resultados obtenidos por dos grupos de alumnos, los que han seguido la formación presencial y los que la han recibido virtualmente. Las conclusiones presentan resultados desfavorables para el grupo que ha seguido el tutorial, lo que lleva a los autores a concluir que el método más eficaz de formación consiste en vincular el tutorial a una clase preparatoria, evidenciando además la necesidad de contar con el apoyo de un bibliotecario para resolver dudas. Otra investigación (Kendall, 2005) presenta un estudio longitudinal de los resultados de dos cursos académicos, en el que se evalúa lo que han aprendido los alumnos sobre modelos de citación bibliográfica. Aunque las conclusiones aportadas son escasas se cree que el tutorial ha tenido un gran impacto sobre el aprendizaje y ha facilitado la mejor integración de los materiales didácticos en el entorno virtual. La misma autora explica una experiencia similar utilizando la misma metodología, en este caso aplicada al tutorial Internet detective (Kendall; Booth, 2003). La principal conclusión del estudio es que el tutorial fue bien aceptado, si bien las encuestas sugirieron algunos cambios para su mejora.

En España las aportaciones sobre el tema siguen la línea de lo ya descrito, pero son escasas y se centran en proyectos de diseño de tutoriales y en el estudio de caso. La primera de ellas es la de Ribes-Llopes (1996) que describe el tutorial multimedia Sirio (Sistemas de Recuperación de la Información) elaborado por la Universidad Politécnica de Valencia (UPV). De-la-Torre-Bustamante (2000) considera que Sirio está más orientado al profesional que al usuario final sobre todo en lo referente a la terminología utilizada. Otro artículo posterior compara las prestaciones de Sirio y de Hot copy searching Dialog, un tutorial de Dialog que enseñaba el sistema de comandos de este distribuidor de bases de datos científicas (Bosch; Seguí, 2001). En cuanto a iniciativas para desarrollar tutoriales destacamos la tarea pionera de la Universitat Politècnica de Catalunya (UPC) y sus proyectos Aabib, plataforma de recursos dedicados a la autoformación (Gómez-Enrich et al., 2002), y FIBU (Formación inteligente en las bibliotecas de la UPC) (Méndez-Planell et al., 2002). Estos esfuerzos han culminado con la adaptación del tutorial TILT (Texas information literacy tutorial) y la creación de uno propio para la consulta del catálogo (Tutorial de consulta del catàleg de les biblioteques de la UPC).

Sin embargo, son aún más escasas las publicaciones que describen un conjunto significativo de tutoriales. Una excepción la constituye el trabajo de Sastre-Miralles (2000), en el que analiza los tutoriales y guías difundidos a través de la web por 44 bibliotecas universitarias españolas. Aunque sus conclusiones son muy generales, el autor hace una serie de recomendaciones para mejorarlos, como la inclusión de un índice de materias y otro alfabético para facilitar la localización de las bases de datos. Más recientemente se ha publicado un artículo que establece un modelo evaluativo de la usabilidad de los materiales formativos virtuales (Marzal et al. 2008). Los criterios para la evaluación se establecen a partir de las aportaciones de la alfin y del constructivismo educativo. El modelo fue contrastado en la práctica a partir de las respuestas de 364 alumnos de primaria y secundaria, los cuales evaluaron la usabilidad de dos recursos educativos en red: Guido contra el señor de las sombras, del Museo 
Thyssen Bornemisza, y Experimentar, del Ministerio de Educación, Ciencia y Tecnología argentino.

De lo expuesto hasta ahora se deduce que no existen estudios que aporten conclusiones en profundidad sobre un número representativo de tutoriales universitarios, ni en menor medida, que establezcan una metodología para evaluarlos.

Se ha partido de una revisión bibliográfica, cuyo análisis ha proporcionado ejemplos de buenas prácticas

\section{Objetivos y metodología}

El objetivo principal de este trabajo es presentar un método de evaluación y diseño de los tutoriales web basado en un conjunto de indicadores. El estudio se acompaña de ejemplos de buenas prácticas que ilustran y describen cada indicador, y que resultan de utilidad para los centros que quieran desarrollar algún tutorial propio o deseen mejorar el actualmente existente. Aunque se enseñan ejemplos de buenas prácticas de tutoriales de bibliotecas universitarias creemos que pueden ser útiles y extrapolables a cualquier tipo de unidad de información.

Para el establecimiento de los indicadores se ha partido de la revisión bibliográfica especializada en ciencias de la educación y documentación, y el posterior análisis nos ha proporcionado los ejemplos de buenas prácticas. El interés se centra en la usabilidad e interactividad de las interfaces de consulta y en los criterios de selección de los contenidos de las páginas web. Estos indicadores se han contrastado con tutoriales actualmente en funcionamiento, lo cual nos ha facilitado localizar buenos ejemplos de cada uno de ellos. Esté método ha sido validado en el estudio descriptivo-analítico de 180 tutoriales universitarios internacionales (Somoza; Abadal, 2009), en la tesis Análisis de los tutoriales web creados por bibliotecas universitarias (Somoza, 2009); y en la descripción de materiales didácticos elaborados en las bibliotecas universitarias españolas en la que se incluye también el estudio de tutoriales (Somoza; Abadal, 2009).

En líneas generales estos indicadores parten de un modelo instructivo basado en unos parámetros básicos que se caracterizan por: incrementar la interactividad entre sistema (interfaz y sistema docente) y alumno; diversificar los conocimientos y maneras de aprender; y ceder todo el protagonismo al alumno para que sea autónomo y responsable en su proceso de aprendizaje, mientras que el tutorial sólo debe proporcionar herramientas que faciliten y garanticen dicho proceso (Varela-Prado, 2009).

En la tabla 1 presentamos los indicadores con una breve descripción de cada uno.

\section{Indicadores y ejemplos de buenas prácticas}

\section{Aspectos descriptivos}

En este apartado los indicadores se refieren a directrices vinculadas a criterios de calidad de las páginas web como son: indicación de la fecha de creación, de actualización e información sobre la autoría y el proceso de creación. Todos ellos aportan información contextual y de utilidad para el usuario que consulta el tutorial. La mayor parte de los tutoriales web no incluyen este tipo de información. He aquí algunos buenos ejemplos:

Pilot, your information navigator, de la Queensland University of Technology Brisbane, Australia https://pilot.library.qut.edu.au/help/about/

LOBO (Library online basic orientation), de las bibliotecas de la North Carolina State University http://www.lib.ncsu.edu/lobo/template.php\#about

Otro aspecto a valorar es la existencia del tutorial en otros idiomas. Cuando la lengua original del tutorial no es el inglés, en muy pocos casos se ofrece la versión en dicho idioma. Una excepción la constituye:

Søk \& Skriv, de la Universitetet I Bergen, Noruega http://www.sokogskriv.no/english/

\section{Contenidos}

Se describe la representación de los contenidos (textuales e imágenes). En la mayoría de los casos se observa con claridad la evolución experimentada en la presentación de contenidos textuales a hipermedia. Los primeros solían tener una extensión superior a lo que se puede leer por pantalla, siguiendo el ejemplo de otras páginas web. La tendencia actual es elaborar pocos contenidos de texto ya que las imágenes $o$ el sonido permiten incrementar la interactividad y hacen que sean más atractivos para el alumno. Algunos buenos ejemplos se encuentran en:

You quote it, you note it!, de la Vaughan Memorial Library, Acadia University, Canadá http://library.acadiau.ca/tutorials/plagiarism/

RIOT (Research information online tutorial), de la Rutgers University, New Jersey http://www.libraries.rutgers.edu/rul/lib_instruct/riot/

En relación con la organización y extensión de los contenidos se constata una tendencia clara hacia la estructura modular porque permite ordenar de una manera más lógica la información contenida sobre todo en aquellos casos en los que el tutorial contiene gran cantidad de ella. De cara al usuario este tipo de estructuración facilita la reanudación de la consulta en caso de abandono. Un ejemplo ilustrativo es:

Smart searcher, de la Deakin University, Melbourne, Australia http://www.deakin.edu.au/library/tutorials/webpac/

En relación con las temáticas, comentar que en la actualidad se observa una doble tendencia: por un lado, los tutoriales más extensos, organizados modularmente y que basan sus contenidos en las competencias informacionales relacionadas con la alfin y las normativas internacionales ( $A L A / A C R L$, Sconul y Anziil); por otro, aquellos centros que prefieren producir tutoriales de una temática concreta con un mayor grado de profundidad de los contenidos. He aquí una muestra de ambas tendencias:

What makes a journal scholarly, de las Rutgers University libraries 


\begin{tabular}{|c|c|c|}
\hline & Indicador & Descripción \\
\hline \multirow{5}{*}{ Aspectos generales } & Idioma & Dispone de versiones en otros idiomas \\
\hline & Fecha de creación & Consta la fecha de creación \\
\hline & Fecha actualización & Consta la fecha de actualización \\
\hline & Background & Explica el proceso de creación \\
\hline & Autoría & Especifica las personas responsables de la creación del tutorial \\
\hline \multirow{2}{*}{ Contenidos } & Tipología & Cómo se representan los contenidos: texto, imágenes, etc. \\
\hline & Estructuración & Organización de los contenidos (modular o lineal) \\
\hline \multirow{4}{*}{ Aspectos docentes } & Ejemplos & Presencia de ejemplos que vinculan la teoría con la práctica \\
\hline & Glosario & Incorpora un glosario \\
\hline & Enlaces externos & Incluye enlaces que complementan los contenidos \\
\hline & Método docente & Didáctica de los contenidos: demostración guiada, resolución de problemas \\
\hline \multirow{2}{*}{ Evaluación del alumno } & Ejercicios & Presencia y tipo de ejercicios: cuestionario, prácticas, juegos, etc. \\
\hline & Valoración de los ejercicios & Existe alguna forma de evaluación o de feedback de los ejercicios \\
\hline Asistencia & Contacto con el bibliotecario & Se pueden plantear dudas o comentarios \\
\hline Evaluación del tutorial & Sistema de evaluación del tutorial & El usuario puede opinar sobre los contenidos y sugerir mejoras \\
\hline \multirow{5}{*}{ Tecnología } & Código de entrada & Identificador del alumno para su mejor seguimiento \\
\hline & Lenguaje de programación & Lenguaje de programación: php, html, etc. \\
\hline & Descarga plugins & Requiere la descarga de plugins \\
\hline & Distintas versiones & Posibilidad de consultar el tutorial sin animación, texto básico, etc. \\
\hline & Derechos & Especifica algún tipo de derecho \\
\hline \multirow{4}{*}{$\begin{array}{l}\text { Autonomía del proceso } \\
\text { de aprendizaje }\end{array}$} & Indicación de objetivos & Presentación de objetivos formativos \\
\hline & Pretest & Inclusión de algún sistema para evaluar los conocimientos previos \\
\hline & Temporalización & Indicación del tiempo estimado para cursar cada uno de los apartados \\
\hline & Sumario & Existe un resumen de los principales contenidos al final de cada módulo \\
\hline \multirow{3}{*}{$\begin{array}{l}\text { Alumnos con } \\
\text { conocimientos } \\
\text { diferentes }\end{array}$} & Niveles & $\begin{array}{l}\text { Incorpora distintos niveles de conocimiento para diferentes usuarios (general, } \\
\text { avanzado, etc.). }\end{array}$ \\
\hline & Perfil de usuario & Especifica el tipo de usuario al que va destinado \\
\hline & Formación a distancia & Incluye información específica para este tipo concreto de usuario \\
\hline \multirow{4}{*}{ Navegación y diseño } & Mapa del sitio & Incluye este elemento \\
\hline & Barra de situación & Incluye este elemento \\
\hline & $\begin{array}{l}\text { Personalización de la pantalla y } \\
\text { colores }\end{array}$ & Existe alguna forma de personalización \\
\hline & Cambio de tamaño letra o contraste & Posibilita opciones de cambio de letra o contraste de la pantalla \\
\hline \multirow{3}{*}{ Interactividad } & Animaciones & Incluye algún tipo de animación \\
\hline & Elementos multimedia & Utilización de audio y vídeo \\
\hline & Contextos didácticos & Incorpora algún escenario que facilite la interacción con el tutorial \\
\hline
\end{tabular}

Tabla 1. Principales indicadores analizados

http://www.rci.rutgers.edu/ estec/tutorials/scholarly.htm

Pilot, your information navigator (ya citado).

\section{Muchos tutoriales se encuentran en una} fase inicial, sin cumplir los requisitos mínimos

\section{Aspectos docentes}

En este apartado se analizan los recursos del sistema para llevar a cabo la formación. En primer lugar es importante conocer el método docente empleado, lo cual no difiere de manera significativa. La mayoría de los tutoriales consultados aplican el método expositivo con ejercicios, seguido de los que utilizan las demostraciones guiadas. Éstas últimas se revelan especialmente útiles para enseñar de manera muy pautada, por ejemplo, cómo buscar información en un recurso concreto. No hemos localizado, en cambio, ningún tutorial que se valga de metodologías docentes innovadoras basadas en la resolución de problemas o en el aprendizaje por descubrimiento. No obstante, alguno de los examinados parte de situaciones o problemas reales como punto inicial de la explicación.

Bruin $^{1}$ success with less stress, de la UCLA Library, Los Ángeles.

http://www.library.ucla.edu/bruinsuccess/

Liberation: using the Web, de la University of Northamptom, Reino Unido

http://library.northampton.ac.uk/liberation/web/index.php

ERIC: an online tutorial, de la Ball State University, Muncie, Indiana

http://www.bsu.edu/libraries/collections/instsvs/eric/ERI Conline.html

Otro aspecto a considerar es la incorporación de ejemplos para ilustrar las explicaciones, los cuales están presentes en prácticamente la totalidad de los tutoriales consultados. La mayoría de ellos incluyen los ejemplos junto al texto para complementarlo. No es el caso del tutorial elaborado por la biblioteca de la Concordia University que separa claramente los ejemplos de los conceptos: 
Info research 101: surviving your essay, de las Concordia University Libraries http://library.concordia.ca/help/tutorial/ILlauncher.htm

A pesar de que el mejor tutorial sería aquél que ofreciera ejemplos de diferentes especialidades para que el usuario pudiera escoger la temática más afín a sus intereses y necesidades, hoy en día existen pocos tutoriales web que incorporen esta opción, excepción hecha de:

InfoSphère, de la Université du Québec à Montréal

http://www.bibliotheques.uqam.ca/ InfoSphere/

Para motivar al usuario a hacer uso del

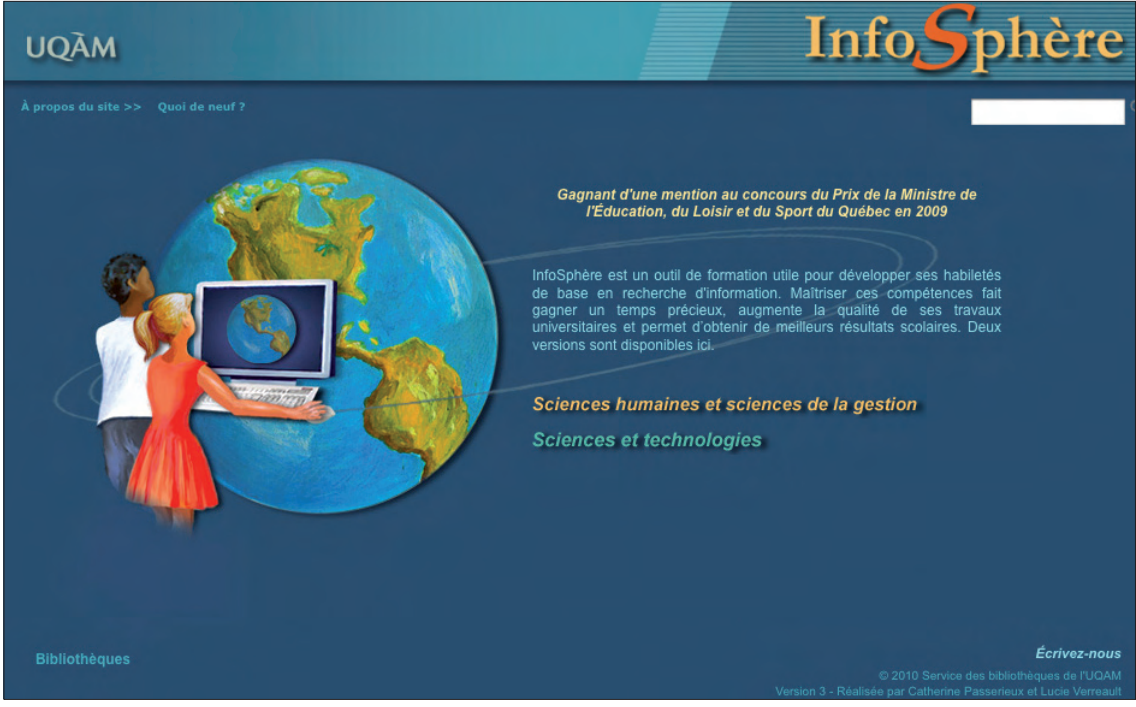

Figura 1. Tutorial con ejemplos de diferentes temáticas (InfoSphère) tutorial, se acostumbra a usar un tono más bien informal y así hacérselo más cercano:

Tutorial en cerca d'informació, del crai de la Universitat de Barcelona

http://www.bib.ub.edu/atri/tutorial1/

Algunos tutoriales incorporan también opciones adicionales como un glosario de términos con el fin de complementar los contenidos. Como recomendación general la bibliografía sobre este tema sugiere no abusar demasiado de la terminología especializada porque puede provocar rechazo y abandono. Para obviar esta dificultad hay tutoriales que ordenan alfabéticamente el glosario con independencia del contenido; mientras que otros presentan la definición desde el propio texto mediante la abertura de una ventana aparte. Sirva como ejemplo de esto:

Information literacy tutorial, de los Five Colleges of Ohio http://collaborations.denison.edu/ohio5/infolit/

Otro asunto importante es la inclusión de enlaces externos a otros recursos de internet para complementar los contenidos. La mayoría de los tutoriales no resuelven bien el tema de la navegación ya que a veces es difícil volver al punto de partida. Algunos solucionan este problema incorporando de manera interna dichos recursos.

\section{Evaluación de los alumnos}

Este indicador se refiere a las opciones ofrecidas por el tutorial para que el usuario autoevalúe lo aprendido. La tipología de ejercicios es variada pero, evidentemente, todos intentan reforzar los conocimientos. En la mayoría de los casos la fórmula más empleada son los cuestionarios que se añaden al final o al terminar un módulo o apartado concreto. En algunos casos se detallan y calculan los aciertos y errores para determinar de una manera más concreta si se supera la formación:
Search path, de la Western Michigan University http://www.wmich.edu/library/searchpath/module.html

Otros, en cambio, presentan ejercicios interactivos en forma de juegos para conseguir mayor implicación o motivación del usuario:

Credible source count, de la Vaughan Memorial Library, Acadia University, Canadá http://library.acadiau.ca/tutorials/webevaluation/

Doing research: An introduction to the concepts of online searching, de la University of Illinois at Chicago http://www.uic.edu/depts/lib/reference/services/tutorials/ Tutorial--Final\%20Version.swf

En el siguiente, por ejemplo, se trata de cuidar a una mascota. A medida que la citación se construye correctamente el animal está bien cuidado y sano:

Liberation: Referencing tutorial, de la University of Northampton, Reino Unido http://library.northampton.ac.uk/liberation/ref/

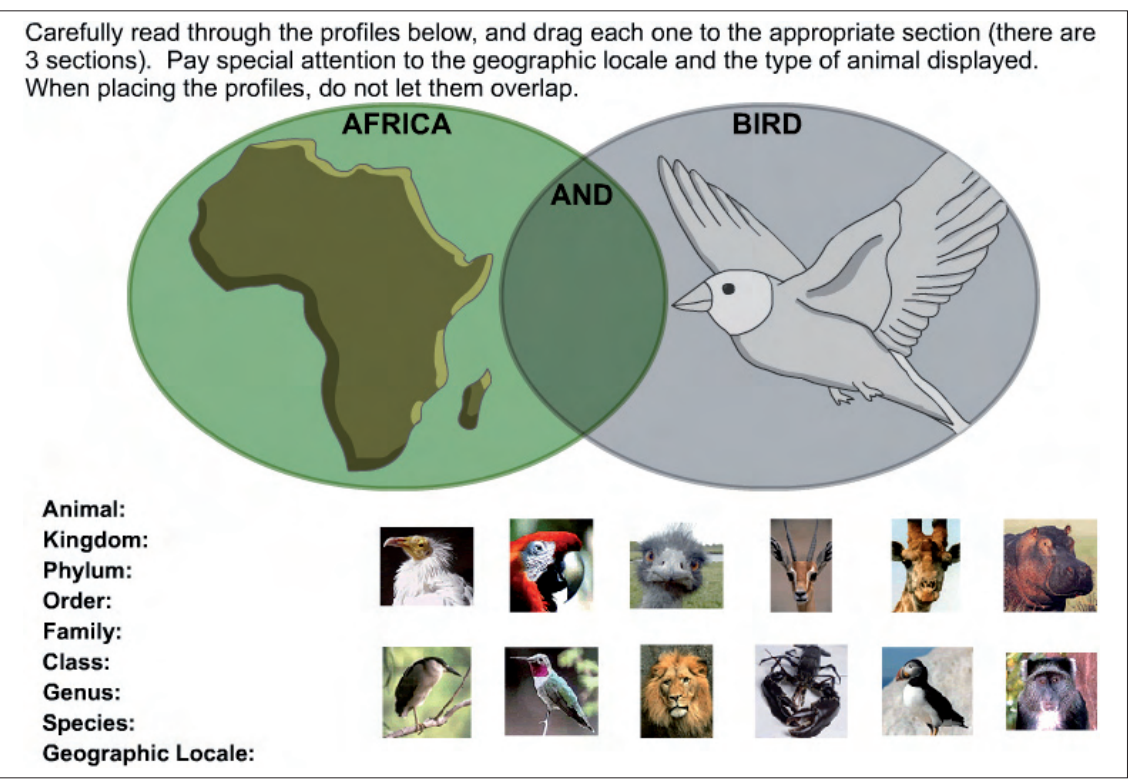

Figura 2. Ejercicio interactivo en forma de juego (Doing research: an introduction to the concepts of online) 
Es importante que aparte de la valoración, los ejercicios incorporen algún tipo de retroalimentación positiva y/o negativa que oriente al alumno.

\section{Asistencia}

En este apartado se valora que el tutorial incluya sistemas que permitan el contacto directo entre sus responsables y los alumnos para que éstos planteen dudas o sugerencias. Cuando incorporan esta prestación la mayoría utiliza chat, teléfono, o correo electrónico, que es la vía de comunicación más habitual. Sin embargo, actualmente una parte considerable de los tutoriales consultados no incluye ningún método de asistencia al usuario.

Uno de los pocos ejemplos de uso del chat como sistema de asistencia lo constituye el OSU Libraries research tutorial, de las Oregon State University Libraries

http://osulibrary.oregonstate.edu/instruction/tutorials/

\section{Evaluación}

Otro indicador complementario es la incorporación de herramientas evaluadoras. Se trata de un sistema para fomentar las mejoras y detectar problemas sobre los contenidos y los aspectos formales y estructurales a partir de la información aportada por los usuarios y que no supone necesariamente cambios en los contenidos ni en la estructura.

La evaluación se materializa mediante encuestas, formularios abiertos o enlaces al correo electrónico. A pesar de que su presencia debe valorarse muy positivamente, la mayor parte de los tutoriales consultados no incorpora ningún sistema de evaluación excepto:

InfoSkills, de la University of Newcastle Library, Reino Unido

http://www.newcastle.edu.au/service/library/tutorials/ infoskills/index.htmly

Pilot, your information navigator (citado anteriormente).

\section{Indicadores tecnológicos}

Este indicador valora que los tutoriales no necesiten descargar complementos o plugins, y, en caso contrario, sería el mismo tutorial el que debería ofrecer la posibilidad de descargarlo. Algunos contemplan la creación de dos versiones: la textual, apta para la impresión y otra más interactiva. Sirvan de ejemplo:

Discus (Developing information skills \& competence for university), de la University Library, Hamburg Institute of Technology

http://discus.tu-harburg.de/login. php?la=engly

CLUE Tutorial, de la University of Wisconsin-Madison

http://clue.library.wisc.edu/

En algunos casos el usuario debe introducir un código personal, lo cual permite registrar su evolución. Mayoritariamente se utiliza para diferenciar al usuario alumno del invitado. De esta manera se asignan más funciones y prestaciones al primero, como la de guardar la puntuación de los ejercicios, mientras que al invitado sólo se le permite la consulta:

Road to research, de la University of California, Los Ángeles http://www.sscnet.ucla.edu/library/index.php

Safari (Skills in accessing, finding and reviewing information), de la Open University Library

http://www.open.ac.uk/safari/

Respecto a la posibilidad de ofrecer los contenidos del tutorial en acceso abierto, hemos de reconocer que son muy pocos los que disponen de licencia Creative commons o de Open publication license, a pesar de ser una muy buena opción en aquellos casos en los que la biblioteca no dispone de medios para desarrollar un tutorial propio:

Bruin ${ }^{1}$ success with less stress (citada anteriormente)

Research 101 (University Libraries of Washington) http://www.lib.washington.edu/uwill/research101/

\section{Autonomía del proceso de aprendizaje}

En este apartado se describen los elementos que favorecen la enseñanza autónoma a fin de que el tutorial se adapte al perfil del máximo número de alumnos. Para que estos se responsabilicen de su proceso de aprendizaje, el tutorial debe proporcionarles herramientas de control que les permitan saber qué aprenderán, en qué momento se encuentran del proceso, qué han aprendido y, en el caso de que conozcan algo de la materia, a partir de qué punto deben iniciar su formación.

El pretest es muy interesante ya que orienta y evalúa los conocimientos o prácticas previas del alumno y le indica qué secciones o módulos ha de consultar. La mayoría de los tutoriales han sido diseñados con la idea de que todos los alumnos saben lo mismo y aprenden de la misma manera. El número de tutoriales que incorporan pretest es escaso todavía y en los pocos que lo presentan, el cuestionario es el formato más extendido. Algunos de ellos además muestran el número de preguntas acertadas e indican qué secciones deben ser consultadas y cuáles no:

Comprehensive online research education, de la Purdue University, Indiana, EUA

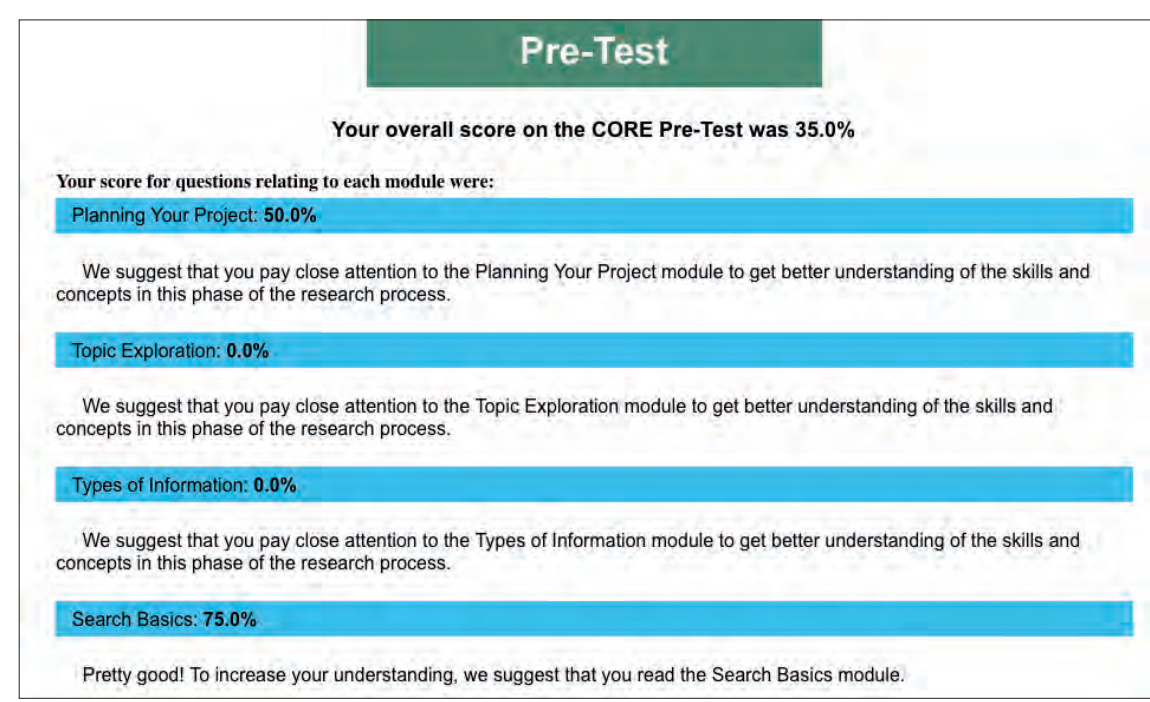

Figura 3. Pretest (CORE tutorial) 
http://gemini.lib.purdue.edu/core/ login/login.cfm

Road to research (citado anteriormente)

La sumarización de contenidos permite hacer una síntesis de lo más relevante que se ha explicado y en algunos casos avanza lo que se explicará a continuación. Se trata de otro elemento más que aporta información sobre el proceso de aprendizaje y muy recomendable en aquellos tutoriales de extenso contenido:

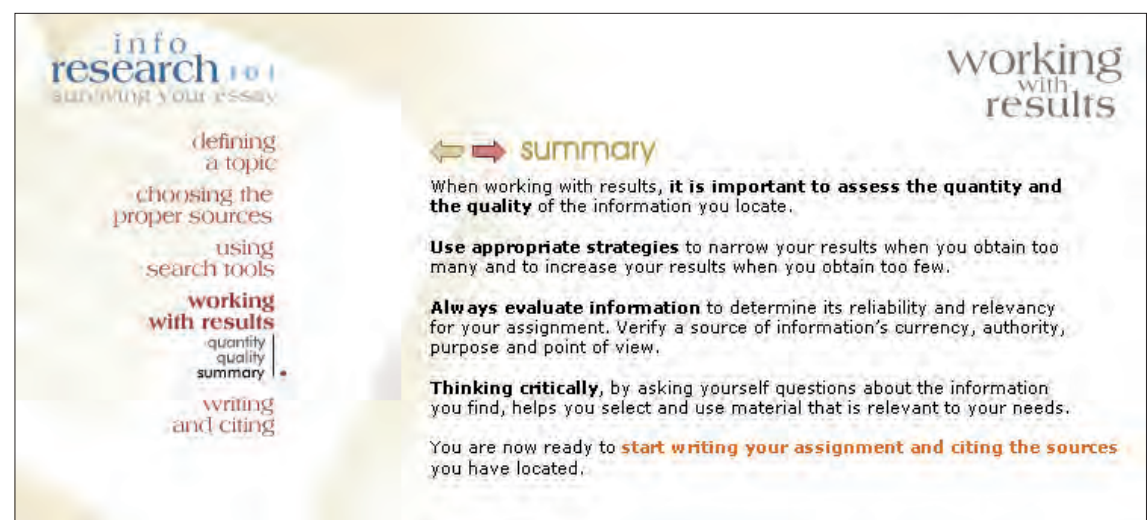

Figura 4. Sumario (Info research 101: surviving your essay)
VIKO Guide to information literacy, de la Norwegian University of Science and Technology

http://www.ub.ntnu.no/viko/en/mod5/mod5_side11.php

Info research 101: surviving your essay, de las Concordia University Libraries, Canadá

http://library.concordia.ca/help/tutorial/ILlauncher.htm

Otro indicador interesante es el de la temporalización, que informa del tiempo estimado que el alumno deberá emplear en la consulta del tutorial, por módulos o en su conjunto. Este elemento favorece la autonomía del aprendizaje ya que permite su planificación.

La inclusión de los objetivos formativos es también un elemento imprescindible. El tutorial ha de formar y no sólo informar sobre una temática concreta, por ello es vital que difundan los objetivos a conseguir tanto a nivel de conocimientos como de competencias. Dichos objetivos pueden presentarse de manera global para el conjunto del tutorial o bien modularmente si está organizado en secciones:

Skills in accessing, finding and reviewing information (Safari), (citado anteriormente)

Publish, not perish, de las University of Colorado Libraries http://www.publishnotperish.org/intro/index.htm

\section{Alumnos con conocimientos diferentes}

Juntamente con el pretest, los tutoriales deberían implementar prestaciones que contemplen los diferentes niveles de conocimiento de los usuarios para garantizar una formación más completa y compleja. Conseguirlo requiere desarrollar contenidos de nivel avanzado, intermedio y básico para cada una de las temáticas. Sin embargo, esta organización por niveles no resulta ser efectiva en aquellos tutoriales muy extensos o que siguen las normativas de alfin.

En la actualidad son pocos los tutoriales que incorporan niveles diferentes y la tendencia general es a concebir los contenidos para unos alumnos que saben lo mismo y que habrán aprendido lo mismo una vez finalizado el tutorial:

Liberation: using the Web (ya citado)
Health Knowledge Network (HKN) tutorials (University of Alberta Libraries, University of Calgary Library, y University of Alberta Academic Technologies for Learning, ATL) http://www.ucalgary.ca/lib-old/agil/HKN/home/index.htm

Søk \& Skriv (citado anteriormente)

Según se trate de formación virtual o presencial, los contenidos, la orientación y el diseño del tutorial serán diferentes. En la formación a distancia, por ejemplo, se deberá poner más énfasis en las explicaciones sobre las características y la manera de acceder a los servicios, aparte de las explicaciones concretas acerca de cuáles son los recursos y cómo consultarlos. Es escaso el número de tutoriales que contemplen a este tipo de usuarios, y si lo hacen, se limitan a incorporar un módulo específico:

WSU Online distance tour, de la Washington State University

http://www.wsulibs.wsu.edu/Electric/trainingmods/DDP Student_Online_Tour/trainer.html

Online Library Center del University System of Georgia http://www.usg.edu/galileo/skills/

\section{Navegación, usabilidad y accesibilidad}

Sin lugar a dudas, estos indicadores provenientes del diseño de páginas web, son los más conocidos. En este sentido, se recomienda que el tutorial disponga de un mapa del sitio o sitemap para que los alumnos vean claramente cómo se han estructurado los contenidos y puedan acceder más rápida- 
mente a los mismos. Aunque la mayoría de los tutoriales emplean un mapa del sitio textual, el ejemplo siguiente muestra un sitemap visual:

Online Information skills tutorial, de la University of New South Wales, Sidney http://eliseplus.library.unsw.edu.au/ sitemap.htm

Una herramienta que mejora la navegación es la utilización de colores o temas asociados a un módulo concreto. De esta manera el alumno identifica los componentes de cada sección del tutorial por su color:

OLAS Information literacy tutorial, de las Waterford Institute of Technology Libraries

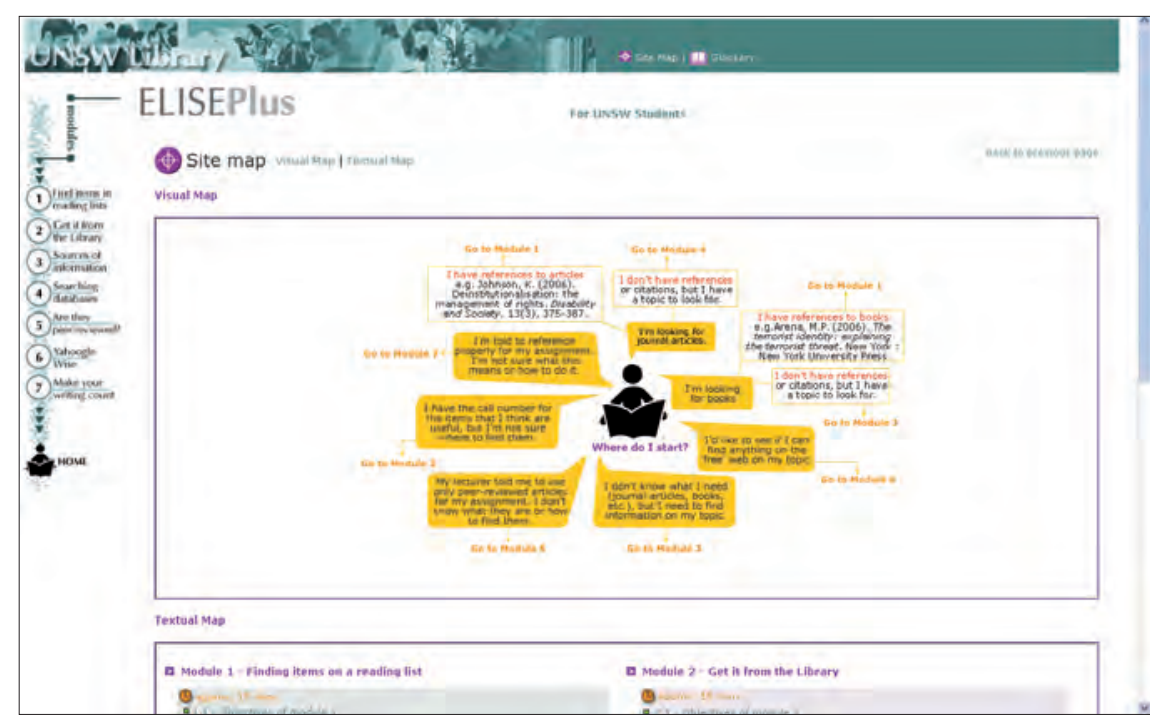

Figura 6. Mapa de sitio visual (Online information skills tutorial) http://library.wit.ie/olas/mod6/start.htm

La barra de situación indicativa de la ruta por la que el usuario ha navegado, se revela como un accesorio útil para situarlo en el contexto de la consulta:

Skils (Skills for knowlegde information \& library searching), de la University of Kent

http://library.kent.ac.uk/skils/index.htm

En lo que respecta a la accesibilidad que permite la personalización de elementos como el tamaño de letra, o el cambio de resolución de la pantalla, por ejemplo, son muy pocos los tutoriales que incorporen estas funciones:

Library induction tutorial, de la University of Bath http://www.bath.ac.uk/library/help/induction/

Liberation: using the Web (citado anteriormente).

\section{Interactividad}

En este punto nos referimos a elementos de animación multimedia que motivan al alumno, presentando el tutorial de una manera más atractiva, aunque existen pocos tutoriales universitarios que los incorporen. He aquí dos ejemplos de buenas prácticas:

Internet detective (Intute virtual training suite) convierte al alumno en un detective que deberá aprender a evaluar la información hallada en internet:

http://www.vts.intute.ac.uk/detective/

La intervención de equipos interdisciplinares encarece el tutorial, pero produce una formación más efectiva

Y en The plagiarism court: you be the judge de la DiMenna-Nyselius Library, de la Fairfield University, Connecticut, el alumno es el juez encargado de juzgar las consecuencias éticas y legales del plagio.

http://www.fairfield.edu/media/flash/library/lib_plagi court.swf

\section{Conclusiones}

El esfuerzo que supone la creación de un tutorial es muy considerable debido al número de variables a contemplar para conseguir crear un recurso formativo que resulte lo más completo y útil posible a sus destinatarios. La mayor complejidad del proceso de aprendizaje universitario exige la creación de tutoriales adaptados al nuevo contexto educativo.

Los indicadores presentados aportan una metodología para evaluar los tutoriales a la vez que brindan herramientas a todos aquellos que quieran crear un tutorial o mejorar las prestaciones del que actualmente ofrecen.

A día de hoy los tutoriales se encuentran en una fase inicial de desarrollo por lo que la gran mayoría de ellos no cumple con los requisitos mínimos, no obstante lo cual, los ejemplos expuestos son muestras de buenas prácticas que orientan también sobre la manera de aumentar su calidad y efectividad.

Las actuaciones más inmediatas a realizar, que no requieren de grandes inversiones ni tecnológicas ni de personal, pasarían por la incorporación de objetivos formativos y de algún tipo de ejercicio práctico a resolver por el usuario. Otras modificaciones como la creación de un pretest y elementos tendentes a favorecer la autonomía del alumno como la sumarización y la temporalización, supondrían la inclusión de mejoras de más amplio calado. Otros cambios más estructurales tales como la incorporación de diferentes niveles o grados de aprendizaje de materias, significaría la desaparición de muchos tutoriales generalistas como es el caso, en 2009, de TILT (Texas information literacy tutorial), uno de los más seguidos y adaptados del mundo.

La diversificación de metodologías docentes y de elementos tecnológicos que favorezcan la interactividad del tutorial representa introducir cambios más profundos, por lo que sería conveniente el apoyo y colaboración de otros especialistas como educadores e informáticos. Si bien es cierto que esta intervención representa un encarecimiento del recurso, la formación de equipos interdisciplinares puede hacer que los tutoriales proporcionen una formación más efectiva. 


\section{Nota}

1. Bruin es un oso pardo, la mascota de la UCLA

\section{Bibliografía citada}

Anderson, Rozalynd P.; Wilson, Steven P.; Livingston, MaryBriget; LoCicero, Allison D. "Characteristics and content of medical library tutorials: a review". Journal of the Medical Library Association, 2008, v. 96, n. 1, pp. 61-63.

Bosch-Pou, Mercè; Seguí-Palou, Rosa. "Productos multimedia aplicados al aprendizaje de las técnicas de recuperación en bases de datos: Sirio y Hot copy searching Dialog tutorial". En: V Congreso ISKO España, Alcalá de Henares: ISKO; Universidad de Alcalá, 2001, pp. 263-271.

Bracke, Paul J.; Dickstein, Ruth. "Web tutorials and scalable instruction: testing the waters". Reference services review, 2002, v. 30, n. 4, pp. 330-337.

Bury, Sophie; Oud, Joanne. "Usability testing of an online information literacy tutorial". Reference services review, 2005, v. 33, n. 1, pp. 54-65.

De-la-Torre-Bustamante, Arantza. "Autoaprendizaje: otra vuelta de tuerca en la formación de usuarios". En: XI Jornadas bibliotecarias de andalucía, Sevilla: AAB, 2000, pp. 465-477.

Dewald, Nancy H. "Transporting good library instruction practices into the web environment: an analysis of online tutorials". The journal of academic librarianship, 1999, v. 25, n. 1, pp. 26-32.

Donaldson, Kelly A. "Library research success: designing an online tutorial to teach information literacy skills to firstyear students". The internet and higher education, 2000, n. 4, pp. 237-251.

Gómez-Enrich, Roser; Méndez-Planell, Montserrat; PérezGálvez, Andrés; Torn-Poch, Pep. "Aabib: La autoformación de usuarios en las bibliotecas de la UPC a través de internet". En: XII Jornadas bibliotecarias de Andalucía, Málaga: AAB, 2002, pp. 367-374.

Hrycaj, Paul L. "Elements of active learning in the online tutorials of ARL members". Reference services review, 2005, v. 33, n. 2, pp. 210-218.

Kendall, Margaret; Booth, Helen. "Developing generic online tutorials as a strategy for extending the use of WebCT". En: Proceedings of 4th Annual conf of the LTSN Centre for Information and Computer Sciences, Galway (Irlanda): LTSNICS, University of Ulster, 2003, pp. 21-26.

Kendall, Margaret. "Tackling student referencing errors through an online tutorial". Aslib proceedings, 2005, v. 57, n. 2, pp. 131-45.
Marzal-García-Quismondo, Miguel-Ángel; Calzada-Prado, Javier; Vianello, Marina. “Criterios para la evaluación de la usabilidad de los recursos educativos virtuales: un análisis desde la alfabetización en información". Information research, 2008, v. 13, n. 4.

http://informationr.net/ir/13-4/paper387.html

Méndez-Planell, Montserrat; Pérez-Gálvez, Andrés; RicoMillán, Oriol; Torn-Poch, Pep. "FIBU: Formación inteligente en las bibliotecas de la UPC". En: III Jornadas de bibliotecas digitales (Jbidi'02), El Escorial (Madrid): JBIDI, 2002, pp. 207-211.

Michel, Stephanie. "What do they really think? Assessing student and faculty perspectives of a web-based tutorial to library research". College \& research libraries, 2001, v. 62, n. 4, pp. 317-332.

Ribes-Llopis, Inmaculada. "Sirio: tutorial multimedia sobre sistemas de recuperación de la información". En: V Jornadas españolas de documentación automatizada, Cáceres: Fesabid, 1996, pp. 847-851.

Sastre-Miralles, Natalia. "Productos y servicios para la formación de usuarios de bibliotecas universitarias: el uso de bases de datos en entornos web". En: VII Jornadas españolas de documentación, Bilbao: Fesabid, 2000, pp. 411-418.

Somoza-Fernández, Marta. Análisis de los tutoriales web creados en las bibliotecas universitarias. Tesis doctoral. Director: Ernest Abadal. Barcelona: Universitat de Barcelona. Departament de Biblioteconomia i Documentació, 2009. http://www.tdx.cat/TDX-0401109-122232

Somoza-Fernández, Marta; Abadal, Ernest. "Analysis of web-based tutorials created by academic libraries". The journal of academic librarianship, 2009, v. 35, n. 2, pp. 126131.

Somoza-Fernández, Marta; Abadal, Ernest. "Evaluación de materiales formativos de acceso público elaborados por bibliotecas universitarias españolas". Revista española de documentación científica, 2009, v. 32, n. 4, pp. 46-66.

Tancheva, Kornelia. "Online tutorials for library instruction: an ongoing project under constant revision". En: ACRL eleventh national conf: learning to make a difference, Charlotte (North Carolina): ACRL, 2003.

http://www.ala.org/ala/mgrps/divs/acrl/events/pdf/tan cheva.PDF

Varela-Prado, Carmen. "Adquisición de competencias en elearning: competencias en información una responsabilidad compartida docencia/biblioteca". En: Ibersid: revista de sistemas de información y documentación. Zaragoza: Prensas Universitarias de Zaragoza, 2009, pp. 303-312. http://eprints.rclis.org/handle/10760/13190 